\title{
HACIA UNA ECONOMÍA POLÍTICA DE LA COMPETENCIA. LA EMPRESA TRANSNACIONAL
}

\author{
Raúl Ornelas ${ }^{1}$
}

Fecha de recepción: 21 de septiembre de 2016. Fecha de aceptación: 08 de febrero de 2017.

\begin{abstract}
RESUMEN
En el presente trabajo se establece un diálogo entre tres propuestas de análisis de la competencia entre empresas; así como el papel que en ella juegan instituciones, economías y sociedades involucradas. Estas interpretaciones aportan elementos para enriquecer la economía política de la competencia, tomando como eje el ejercicio del poder que implica la acción de las empresas, en particular la de las grandes corporaciones que modelan el mundo contemporáneo.
\end{abstract}

Palabras clave: competencia económica, empresas transnacionales, cadenas globales de valor, costos de transacción, teoría de la empresa.

Clasificación JEL: D23, D46, F23, K21, L41.

\section{Towards a Political Economy of Competition: Transnational Companies}

\begin{abstract}
This paper strikes up a dialogue among three approaches to parsing competition between companies; it also examines the role in competition played by institutions, economies, and the societies involved. These interpretations provide the building blocks to enrich the political economy of competition, predicated on the exercise of power entailed by the actions companies take, in particular, the large corporations that shape the contemporary world.
\end{abstract}

Key Words: economic competition, transnational companies, global value chains, transaction costs, business theory.

${ }^{1}$ Instituto de Investigaciones Económicas, Universidad Nacional Autónoma de México (UNAM), México. Correo electrónico: raulob@iiec.unam.mx 
VERS UNE ÉCONOMIE POLITIQUE DE LA COMPÉTENCE. L'ENTREPRISE TRANSNATIONALE

\section{Résumé}

Dans le présent document, on établit un dialogue entre trois propositions de l'analyse de la compétence entre les entreprises ; ainsi que le rôle qui jouent des institutions, des économies et des sociétés impliquées. Ces interprétations apportent des éléments pour enrichir l'économie politique de la compétence, en prenant comme axe central l'exercice du pouvoir que l'action des entreprises implique, en particulier, celle des grandes corporations qui modèlent le monde contemporain.

Mots clés: compétence économique, entreprises transnationales, chaînes globales de valeur, coûts de transaction, théorie de l'entreprise.

\section{PARA UMA ECONOMIA POLÍtica DA CONCORRÊNCIA. A EMPRESA TRASNA- CIONAL}

\section{Resumo}

No presente artigo se estabelece um diálogo entre três propostas de análises da concorrência entre empresas; bem como o papel que nela jogam instituiçóes, economias e sociedades envolvidas. Estas interpretaçóes contribuem com elementos para enriquecer a economia política da concorrência, tomando como eixo o exercício do poder que implica a ação das empresas, em particular a das grandes corporaçóes que modelam o mundo contemporâneo.

Palavras-chave: concorrência econômica, empresas multinacionais, correntes globais de valor, custos de transação, teoria da empresa.

跨国公司的竞争力政治经济学

劳尔・奥尔奈拉斯

摘要

本文通过三份关于公司间竞争的分析，透视在公司的竞争中相关政府机 构、经济体和企业所起的作用。在分析中本文以公司的行为、尤其是对当 代世界有重大影响的国际大型企业的行为作为出发点。本文的阐释将为竞 争力政治经济学的丰富和发展略尽绵薄之力。

关键词: 经济竞争跨国公司全球价值链交易成本 


\section{INTRODUCCIÓN ${ }^{2}$}

Desde los años ochenta del siglo xx, la economía mundial vive una profunda transformación, tanto en las relaciones que la articulan como en los actores que orientan su trayectoria. De la mano de las reformas regulatorias impulsadas por gobiernos conservadores, las grandes empresas se convirtieron en los sujetos dominantes del mercado mundial, desplazando el protagonismo de los Estados, cuyas funciones devinieron subsidiarias de las necesidades, proyectos y estrategias empresariales. Esta transformación ha dado lugar a una gran cantidad de teorías y análisis que pretenden explicar la actividad empresarial.

En el presente trabajo se establece un diálogo entre tres propuestas de análisis de la competencia entre empresas, así como el papel que en ella juegan instituciones, economías y sociedades involucradas. Estos enfoques aportan algunos elementos básicos para enriquecer la economía política de la competencia, tomando como eje el ejercicio del poder que implica la acción de las empresas, en particular la acción de las grandes corporaciones que modelan el mundo contemporáneo.

Del extenso repertorio de teorías sobre la empresa, destacamos dos que por su alcance y complejidad dibujan las dinámicas y las dimensiones esenciales de la competencia: la teoría de los costos de transacción y la teoría de las cadenas de valor. Hemos elegido estas teorías en tanto constituyen el principal paradigma de análisis de las empresas, tanto para la academia como para las instituciones multilaterales y el propio mundo empresarial. Tras exponer sus principales planteamientos, las relacionamos con algunos aportes del pensamiento crítico acerca de la internacionalización del capitalismo. ${ }^{3}$

\section{PARADIGMAS DE LA TEORÍA DE LA EMPRESA}

En los años noventa del siglo $\mathrm{xx}$, los estudios sobre la actividad empresarial delimitaban dos grandes grupos de interpretaciones: aquellas centradas en el "poder de mercado" y las fundadas en la internalización de los costos de transacción (Cantwell, 1991). Ambos cuerpos derivan de la teoría económica neoclásica y comparten el punto de partida de considerar a la empresa como un agente individual.

\footnotetext{
Investigación realizada gracias al Programa UNAM-DGAPA-PAPIIT IN302215.

Las principales teorías de la firma las podemos encontrar en Pitelis y Sugden (2000); Coriat y Weinstein (2011); Foss (2000).
} 
Las teorías del poder de mercado (Kindleberger, 1969; Hymer, 1976) analizan las formas de organización de las empresas y su capacidad para controlar los mercados en que actúan, así como las consecuencias que la acción de las transnacionales tiene para las economías anfitrionas: limitación de la competencia, desequilibrio de la balanza de pagos y pérdida de soberanía, entre las más importantes.

Las teorías de la internalización de los costos de transacción priorizan el análisis de la eficiencia económica y conciben a las empresas como instituciones que permiten aumentar la riqueza y el bienestar social; en sus desarrollos más completos, estas teorías destacan el papel crucial que juega el ambiente institucional en que actúan las empresas para lograr resultados óptimos en la asignación de los recursos productivos.

Este panorama teórico y analítico cambió como resultado de la evolución de la economía mundial. La expansión planetaria de las empresas representó el fin de los nacionalismos económicos que caracterizaron a los capitalismos metropolitanos desde la Revolución Industrial: la internacionalización alcanza un punto de inflexión con la reconstrucción de Europa y Japón después de la Segunda Guerra Mundial y con la creciente industrialización de las semiperiferias en Asia y América Latina. La formalización de estos procesos de interpenetración económica dio fuerza al estudio de la eficiencia de las empresas: si el crecimiento económico no dependía más de los Estados, era necesario conocer a fondo las condiciones en que las empresas pueden generar riqueza y mayor bienestar para las sociedades. Ello propició importantes avances de las disciplinas que estudian la actividad empresarial: sociología, economía, geografía, y particularmente la teoría de la empresa.

Durante los ańos noventa del siglo pasado, se produce un viraje radical en el paradigma del análisis sobre las empresas transnacionales. El enfoque de los costos de transacción toma el relevo del enfoque estructuralista, anclado en los efectos del ejercicio del poder sobre las economías nacionales, característicos de la actividad empresarial.

Ello expresa el profundo clivaje en el conjunto de la vida social hacia una nueva legitimidad del capitalismo. La presencia de las empresas transnacionales dejó de ser considerada como un peligro para la integridad y el desarrollo de los territorios en los que se alojan, convirtiéndose en uno de los principales indicadores de la confianza que los mercados tienen en las economías que reciben las inversiones transnacionales. Se transita de una actitud centrada en la regulación de las empresas hacia políticas de estímulo a la captación de inversiones extranjeras: su atracción deviene un objetivo estratégico de la intervención del Estado. 


\section{Los costos de transacción y las instituciones del capitalismo}

En este apartado presentamos una síntesis sobre el enfoque que constituye el paradigma actualmente dominante en el estudio de las empresas transnacionales: la teoría de la internalización de los costos de transacción. Este enfoque toma como punto de partida la teoría neoclásica e intenta superar algunos aspectos poco desarrollados de la misma, como es el caso de la teoría de la firma, la dinámica de la innovación y el papel de las instituciones.

El concepto de internalización de los costos tiene su origen en el trabajo de Coase, titulado The Nature of the Firm (1937), en el que plantea que la racionalidad de la empresa, en tanto institución económica, es la reducción de los costos de las transacciones económicas; la empresa es considerada como un agente de la eficiencia y creadora de riqueza.

En Las instituciones económicas del capitalismo (1989), Williamson desarrolla el marco teórico de la economía institucional, que propone un análisis en términos de gobernanza, buscando abarcar al conjunto de actividades realizadas por las empresas y su entorno económico y social: "Una característica común de la nueva línea de investigación es que el concepto de la empresa como una función de producción se ve sustituido (o incrementado) por el concepto de la empresa como una estructura de gobernación" (Williamson, 1989: 26).

El comportamiento de las empresas está regido por la internalización de los costos de transacción. Al incorporar ciertas transacciones mediante su organización, la empresa reduce los costos del funcionamiento económico. La preocupación central de este enfoque es explicar las formas de organización económica alternativas al mercado, como son la jerarquía y el contrato. A partir de los análisis sobre las "fallas del mercado", se critica el planteamiento neoclásico de que todas las transacciones económicas hallan una forma de realizarse en los mercados. Frente a la existencia de transacciones que no pueden realizarse a través de los mercados, y de transacciones que son realizadas en forma más eficaz mediante contratos y/o la actividad de las empresas, la economía institucional propone ampliar el territorio del análisis económico. En particular, considera que la internalización de las operaciones por parte de las empresas constituye, a menudo, una solución más eficaz desde el punto de vista de los costos de transacción.

La economía institucional concentra su análisis en los procesos de contractualización que guían el funcionamiento económico. En tanto que las relaciones entre los agentes económicos tienen formas alternativas de ser realizadas 
(operaciones mercantiles, contrato o jerarquía), el análisis busca esclarecer bajo qué condiciones las elecciones de los agentes son las más eficientes.

Este enfoque ha hecho frente tanto a los argumentos de la teoría neoclásica como a aquellos que explican la creación de jerarquías como prácticas monopólicas. Si los primeros son fácilmente rebatibles a través de las actividades de las empresas gigantes, los argumentos en torno de los efectos de monopolio ameritan una discusión amplia. De acuerdo con Williamson:

Todos los enfoques del contrato... tanto los del monopolio como los de la eficiencia, se ocupan de la misma interrogante: ‘a qué propósitos se sirve cuando se sustituye el intercambio de mercado clásico -en el que se vende el producto a un precio uniforme para todos los clientes, sin restricción- por formas de contratación más complejas (incluidos los modos de organización económica ajenos al mercado)? Los enfoques monopólicos imputan las desviaciones de la norma clásica al propósito monopólico. Por otro lado, los enfoques de la eficiencia sostienen que las desviaciones sirven a los propósitos de la economización (1989: 35).

Esta visión del proceso económico se relaciona de manera directa con la teoría de la empresa transnacional: las empresas gigantes encuentran su legitimidad en la eficiencia que logran a través de su organización. La teoría institucional considera que existen tres elementos principales para explicar las prácticas no mercantiles de las empresas: 1) la existencia de una racionalidad limitada, 2) las prácticas de oportunismo, y 3) la especificidad de los activos de las empresas.

Los dos primeros, llamados por Williamson "hipótesis comportamentales”, plantean serios cuestionamientos a las hipótesis básicas de la teoría económica neoclásica, que supone la uniformidad de la información que poseen los agentes económicos y, por tanto, la imposibilidad de aprovechar las asimetrías en ese dominio.

La especificidad de los activos constituye el elemento más importante para explicar la forma que adquieren las "interfaces" de las transacciones: el hecho de que los activos comprometidos sean importantes y peculiares para la empresa que los moviliza, inducirá a las partes involucradas en la transacción a establecer una relación bilateral que les dé un cierto grado de certidumbre, en oposición a las condiciones relativamente más aleatorias que tienen los intercambios mercantiles. Este elemento es, además, esencial para explicar el comportamiento de las grandes empresas, cuyos recursos poseen dimensiones y especificidades particularmente importantes. 
La presencia de esos tres elementos hace necesaria la gobernanza como marco de acción de los agentes económicos y reduce los marcos alternativos (planificación, promesa y competencia), a casos particulares en los que uno o varios de esos elementos principales están ausentes.

\section{Los costos de transacción y la empresa multinacional}

Para la economía institucional, el estudio de la empresa moderna requiere el estudio de la integración vertical, a fin de explicar cómo y en qué circunstancias la acción de las empresas implica objetivos de eficiencia.

En términos históricos, el pasaje de las unidades familiares hacia las firmas gigantes, exigió una transformación radical de las formas de gestión y de organización; la organización multidivisional es uno de los ejemplos más conocidos al respecto.

Williamson considera que la rentabilidad de las empresas y su funcionamiento fueron frenados por tres rasgos de su organización interna: 1) las estructuras fuertemente centralizadas, 2) la incapacidad de los cuadros dirigentes de representar el conjunto de los intereses de la empresa, y 3) los límites que ello implicaba para la asignación eficiente de los recursos.

La estructura multidivisional superó esos obstáculos mediante dos innovaciones cruciales: 1) la creación de una instancia de dirección capaz de elaborar la estrategia general, y 2) dotar de autonomía a las divisiones de la empresa. Esta estructura concedía mayor libertad de acción a las "divisiones operativas semiautónomas" o "centros de beneficios", organizados a partir de criterios como las líneas de producción, la implantación geográfica, los tipos de productos, etcétera, y creaba la "dirección general", encargada de supervisar el desempeńo de las divisiones y formular planes para el conjunto de la empresa. Así surgió una nueva forma de asignación de los recursos: por una parte, los centros de beneficios comenzaron a competir entre sí, eliminando gran parte de los obstáculos burocráticos de las grandes corporaciones centralizadas; por otra parte, la dirección recibió la atribución de sancionar, en última instancia, el reparto de los recursos resultado de dicha competencia intraempresa (Williamson, 1989: 284-285).

La nueva estructura organizativa explica la expansión de las empresas, bajo la forma de la diversificación (creación de conglomerados) y/o de la internacionalización de las operaciones. Así, la empresa multinacional es vista como la entidad económica capaz de gestionar activos localizados en diferentes países (Williamson, 1989: 294). 
La consideración de la organización industrial permite un diálogo fecundo con las interpretaciones críticas que se interesan por los temas de la monopolización, las ganancias extraordinarias y la geopolítica mundial. En efecto, profundizar en los modos en que empresas y gerencia organizan la valorización del capital hace posible un conocimiento puntual sobre las fortalezas y contradicciones de la economía capitalista.

A través del análisis de las transformaciones en la organización interna de las empresas, el enfoque de los costos de transacción intenta demostrar que la diversificación y la integración realizadas por la gran empresa no siempre implican el surgimiento de prácticas monopólicas. La estructura multidivisional constituye un medio para alcanzar una mayor eficiencia en las operaciones de las empresas, en tanto que las prácticas anticompetitivas derivan de las características del medio en que actúan las empresas.

Para la economía institucional, la expansión internacional de las empresas está relacionada con la búsqueda de aumentos de eficiencia. La empresa multinacional busca las mejores "interfaces" para realizar las tres transacciones consideradas como las más importantes para este tipo de firmas: 1) transferencia de capital, 2) transferencia de tecnología, y 3) transferencia de competencias organizativas. De ellas, la transferencia de tecnología es la más problemática; las otras dos son, en principio, realizables al interior de los mercados. En cambio, la transferencia de tecnología implica cuestiones que la hacen impracticable por la vía de los intercambios mercantiles, a saber: la identificación, la divulgación y la formación del equipo que usará la tecnología, una vez instalada en su nuevo emplazamiento.

Si la tecnología es compleja, y si el conocimiento es un elemento central, la compra de este elemento es poco viable, por lo que se impone una inversión en el extranjero. Esto último supone, generalmente, la creación de una jerarquía bajo la forma de una filial que responda a los rasgos referidos de la transferencia de tecnología. La creación de una filial es la solución más eficiente, en tanto que permite un mayor control sobre el gran número de variables que entran en juego en este tipo de transferencias. Por ello, las actividades en las que más se invierte en desarrollo tecnológico son precisamente aquellas que más se internacionalizan.

Este es otro terreno importante de convergencia con los abordajes críticos sobre las empresas transnacionales: el modo de producción capitalista tiene como característica esencial la transformación permanente de sus bases productivas, en particular, de las tecnologías. En tanto medio de elaborar mercancías, la tecnología es también motivo y medio para la internacionalización de 
las empresas. Dado su carácter estratégico, la tecnología tiende a estar fuertemente controlada por las grandes empresas.

En lo que toca a los efectos de monopolización, Williamson aporta argumentos en negativo, tratando de probar que las prácticas anticompetitivas no son resultado directo de las inversiones en el extranjero. En primer lugar, porque el aumento de control de una empresa sobre un mercado dado puede ser realizado mediante transacciones financieras, en especial mediante inversiones de portafolio y no a través de la elaboración de mercancías. En segundo lugar, si la búsqueda de un mayor control de mercado fuera la causa de las inversiones extranjeras, no ocurriría la diferenciación por actividades que implica la internacionalización de las operaciones empresariales. Todas las actividades fuertemente concentradas deberían invertir en el extranjero, lo que no ha sucedido en industrias como la del tabaco, el vidrio y la siderurgia.

Estos son los principales aportes del enfoque de los costos de transacción para el análisis del comportamiento de las empresas transnacionales. Desde nuestro punto de vista, estos aportes acercan la teoría a las realidades económicas contemporáneas. En términos epistemológicos, se argumenta en pro de un análisis meso-económico, enfatizando el papel que tiene la organización y la tecnología como dimensiones esenciales de la actividad empresarial. Esta interpretación no se interesa mayormente en la dimensión mundial del ejercicio del poder: en tanto instituciones económicas, las empresas transnacionales modelan no sólo los mercados, sino también gran parte de las relaciones sociales que articulan el capitalismo. Al situar el análisis en la competencia al interior de las actividades económicas, el paradigma institucional deja fuera la problemática de la formación de coaliciones formadas por empresas y Estados que se disputan los mercados y el poder a escala mundial.

\section{EL PARADIGMA DE LAS CADENAS DE VALOR}

La teoría institucional tiene una de sus principales vías de desarrollo en el análisis de las cadenas de valor, cuyas primeras formulaciones podemos rastrear en la obra de Marshall (2005), que identifica la existencia de concentraciones de ciertas industrias en localidades específicas a las cuales llamó distritos industriales. Otro antecedente de este enfoque son los trabajos sobre competitividad de Porter (1991), quien estableció los factores que permiten determinar los casos exitosos de inserción internacional en los mercados.

Una de las vertientes más actuales e influyentes de este tipo de análisis son los trabajos de Gereffi, quien ha analizado los cambios en la economía mun- 
dial derivados de la industrialización orientada por las exportaciones, y que afectan tanto a los actores como a las regiones que dinamizan el capitalismo. Para este enfoque, los actores predominantes son los agrupamientos de empresas conectados en redes de alcance mundial; asimismo, son ciertas regiones de reciente industrialización, como el Sudeste Asiático y América Latina, que alojan las experiencias más exitosas de tales redes de producción (Gereffi et al., 1994; Gereffi, 2001).

Estos análisis se centran en las formas organizativas típicas del capitalismo contemporáneo, las cadenas de valor integradas regional y mundialmente. En su primera formulación, se propuso el concepto de cadena de mercancías básicas (commodity chain) abarcando tanto las formas en que se integra la producción en diversas escalas geográficas y organizativas gracias a la actividad empresarial, como el establecimiento de jerarquías que permiten la concentración de las decisiones y la apropiación de ganancias (Bair, 2008). Este concepto forma parte de la macro-sociología que estudia el sistema-mundo. Hopkins y Wallerstein (1986: 156) definen la cadena de mercancías básicas como "una red de trabajo y procesos de producción cuyo resultado final es una mercancía básica terminada”.

Este abordaje destaca tres aspectos principales:

1. Las evoluciones de la división internacional del trabajo, mostrando que las cadenas de producción son consustanciales al capitalismo, al menos desde la integración del mercado mundial en el siglo Xvi.

2. La apropiación desigual de los beneficios obtenidos, tomando en cuenta la ubicación de algunos eslabones de la cadena en el centro y otros en las semi-periferias y periferias.

3. Las variaciones en el alcance de las cadenas debidas a las variaciones cíclicas del capitalismo: las fases de contracción conducen a una reducción en el número de participantes, mientras que las fases de expansión tendrían el efecto contrario, aumentar los participantes en las cadenas.

La cadena de mercancías básicas complementa el análisis del sistema-mundo y muestra cómo se articula el proceso de valorización del capital en escalas meso y micro económicas.

Esta perspectiva sobre la organización de las actividades deriva en dos vertientes que se interesan más por el funcionamiento de las cadenas productivas que por su relación con la totalidad capitalista. Gereffi y sus colaboradores han formulado los conceptos de cadenas globales de mercancías básicas (global 
commodity chains-GCC) y cadenas globales de valor (global value chains-GVC), en un intento de superar las limitaciones de un análisis anclado en los bienes finales, ampliando el estudio de la organización productiva hacia las formas de "agregar valor".

La cadena global de mercancías básicas es definida de la siguiente manera:

Una cadena global de mercancías básicas consiste en redes interorganizacionales agrupadas alrededor de una mercancía básica o producto, relacionando hogares, empresas y estados, entre ellos y con la economía-mundo. Estas redes son específicas situacionalmente, construidas socialmente e integradas localmente, destacando la inserción social de la organización económica (Gereffi et al., 1994).

A través de esta conceptualización, se sistematizan estrategias y prácticas de quienes participan en las cadenas de producción, y se establece el carácter de las actividades que hacen posible la elaboración de los productos, tanto en lo que toca a su contenido concreto (adquisición de medios de producción y fuerza de trabajo), como a las formas de despliegue espacial características del capitalismo globalizado.

Las diferencias en las formas y niveles de desarrollo económico son tratadas como temas ligados al "acceso a los mercados y a los recursos". En esta perspectiva, la innovación y la competencia modelan actividades y regiones en el sistema-mundo: las regiones centrales (core) concentran mayores cuotas de riqueza gracias a la producción de innovaciones que, a su vez, transfieren la competencia hacia las regiones periféricas.

Estas consideraciones generales ofrecen un vasto campo de análisis sobre las actividades de las empresas transnacionales. Pensar la producción capitalista como cadenas de mercancías permite destacar la unidad del mercado mundial, estableciendo el papel que juega cada fase en la creación de riqueza. También se destaca el papel del desarrollo científico y tecnológico, en tanto elemento central que articula la producción de mercancías e influye de manera decisiva en la trayectoria de la competencia y en la distribución del excedente.

Sobre la base de la organización productiva se articula el elemento clave de las cadenas de mercancías: las estructuras de gobernanza. Analizando en clave histórica la evolución de la organización de las empresas, Gereffi refiere el tránsito desde la internalización de actividades que caracterizó a las grandes empresas y conglomerados de la producción fordista, hacia la externalización que permite la producción flexible contemporánea, siendo la subcontratación generalizada un ejemplo típico de este proceso. En ese contexto, la 
gobernanza corporativa es esencialmente una estructura de coordinación y no implica necesariamente relaciones de subordinación entre los participantes en las cadenas: se trata de una práctica de integración espacial y productiva y no de una jerarquía.

Este planteamiento inicia el acercamiento a las concepciones y los procedimientos de la economía convencional, mismos que se acentúan en el enfoque de las cadenas globales de valor.

Otro aspecto relevante para el análisis de las actividades de las empresas transnacionales es la caracterización de las cadenas de mercancías según la dinámica que las articula. Las grandes corporaciones actúan en las cadenas globales de mercancías "articuladas por los productores" (producer-driven), debido al uso intensivo de capital y los altos niveles de inversión necesarios para participar en actividades intensivas en capital (automotriz, aeroespacial). Las cadenas "articuladas por el comprador" (buyer-driven) corresponden a las actividades con menores intensidades tecnológicas y en las que predominan las empresas dedicadas a la comercialización y a organizar las redes internacionales de producción, como es el caso de la fabricación de calzado y ropa deportiva.

Esta caracterización también se aplica a las estructuras de gobernanza. Los estudios sobre ambos tipos de cadenas muestran que las estructuras centralizadas son propias de las cadenas articuladas por los productores, en tanto que la descentralización predomina en las cadenas articuladas por los compradores.

Bair (2008) argumenta que la adopción de este enfoque como uno de los principales paradigmas en el análisis del desarrollo económico mundial, está ligada al cambio histórico realizado por los países del Sur global hacia estrategias exportadoras, en las que la posibilidad de alcanzar el desarrollo queda determinada por la capacidad de integrarse en las cadenas globales de mercancías. Este argumento marca una diferencia sustancial respecto de la matriz teórica original del sistema-mundo. En contrapunto a las ideas del intercambio desigual, la dependencia y los efectos de dominación en escala mundial, el enfoque de las cadenas globales de mercancías básicas enfatiza la posibilidad de desarrollar regiones y países mediante la incorporación a las cadenas de producción.

\section{Empresas líderes y cadenas de valor}

Tras su éxito inicial, el enfoque de las cadenas globales de mercancías básicas evoluciona hacia una conceptualización más compleja, bajo el impulso de dos cuestionamientos: 
La referencia a las mercancías básicas (commodities), pues las cadenas de producción también se integran en actividades de gran complejidad tecnológica, lo que obligó a cambiar la denominación original.

El cuestionamiento esencial fue mostrar las limitaciones en el análisis de las estructuras de gobernanza que caracterizaban al enfoque de las cadenas globales de mercancías básicas, pues la articulación de las cadenas no se realiza únicamente por productores o compradores: estudios de caso y debates teóricos llevaron a mostrar el carácter dinámico del papel de productores y compradores, puesto que los avances tecnológicos y su difusión han hecho posible que esos roles sean intercambiables dentro de las cadenas de producción.

Estas evoluciones desembocan en el concepto de cadena global de valor, definida de la siguiente manera:

La cadena de valor describe el rango de actividades realizadas por firmas y empleados para producir un bien o servicio desde su concepción hasta su uso final y más allá. Ello incluye actividades como diseńo, producción, mercadotecnia, distribución y servicios al cliente... Una cadena global de valor está dividida en múltiples empresas y espacios geográficos (Stacey, 2016).

A partir de la oposición entre mercados y jerarquías y del concepto de costos de transacción, Gereffi et al. (2005) elaboran una explicación acerca de cómo se organiza la "fragmentación" de la producción capitalista, caracterizando las cadenas de producción como formas de gobernanza. La gestión de las tres relaciones básicas de la organización productiva, a saber, especificidad de activos, oportunismo y costos de coordinación, puede llevarse a cabo tanto a través de la internalización como mediante una mayor división de trabajo estableciendo relaciones con otras empresas. En ese sentido, las relaciones esenciales para la articulación de las redes de producción no son las de propiedad si no las de coordinación.

De este modo, el enfoque de las cadenas de valor se separa de las preocupaciones derivadas del análisis del sistema-mundo y de las desigualdades productivas, así como de apropiación de la riqueza, para dirigir la atención hacia los temas de la organización y la eficiencia. En ese sentido, constituye un desarrollo de la teoría de la empresa basada en los costos de transacción.

A las relaciones de mercado e integración vertical este abordaje añade el concepto de "red", para dar cuenta de las relaciones de coordinación entre empresas que no pasan por el mercado, proponiendo tres formas de gobernanza: modular, relacional y cautiva. Sobre esa base, el enfoque de las cadenas 
globales de valor ofrece una tipología de las estructuras de gobernanza (Gereffi et al., 2005: 83-84):

1. Mercados. Es la transacción típica de la economía capitalista. Más que la duración de los vínculos, se destacan los bajos costos de cambiar de proveedor o comprador.

2. Cadenas de valor modulares. Los clientes fijan las especificaciones para los proveedores, que conservan importantes márgenes de maniobra merced al uso de tecnologías genéricas y al descargar parte de las inversiones en los compradores.

3. Cadenas de valor relacionales. Son las redes en que existe el mayor intercambio posible entre los participantes, lo que genera situaciones de interdependencia y especialización de los activos cada vez mayores, así como una distribución más equilibrada del poder y los beneficios generados.

4. Cadenas de valor cautivas. Los compradores fijan los términos de las transacciones, fuertemente supervisadas y controladas por las empresas líderes, generando altos costos por cambiar de clientes, por lo que se considera "cautivos" a los productores.

5. Jerarquía. Refiere las prácticas de integración vertical y su principal forma de gobernanza es el control empresarial, a través del cual la administración central transmite sus instrucciones hacia los subordinados, subsidiarias y filiales.

Las relaciones atribuidas a la forma red se alejan del control directo que ejerce la jerarquía empresarial mediante la división del trabajo al interior de la firma, pero son más estables y más codificadas que las transacciones realizadas en los mercados.

A partir de esta tipología, el enfoque de las cadenas de valor establece patrones de gobernanza, poniendo en juego tres tipos de relaciones en el manejo de los elementos necesarios para la producción de mercancías: 1) la complejidad de las transacciones, destacando las especificaciones de productos y procesos; 2) la posibilidad de codificar y transmitir la información sin necesidad de nuevas inversiones; y 3) la capacidad de los proveedores para hacer frente a las demandas de los compradores (Gereffi et al., 2005: 85). A cada uno de estos elementos se le asigna el valor de "alto" o "bajo", dando lugar a las cinco formas de gobernanza típicas de las cadenas globales de valor, que se presentan en el cuadro 1 .

A través de esta formalización, se establecen las relaciones que determinan la posición de las empresas dentro de las cadenas de valor: la coordinación 
Cuadro 1. Principales determinantes de la gobernanza de las cadenas globales de valor

\begin{tabular}{lcccc}
\hline Tipo de gobernanza & $\begin{array}{c}\text { Complejidad de las } \\
\text { transacciones }\end{array}$ & $\begin{array}{c}\text { Habilidad para } \\
\text { codificar las } \\
\text { transacciones }\end{array}$ & $\begin{array}{c}\text { Capacidades de los } \\
\text { proveedores }\end{array}$ & $\begin{array}{c}\text { Grado de } \\
\text { coordinación y } \\
\text { asimetría de poder }\end{array}$ \\
\hline Mercado & Baja & Alta & Alta & Baja \\
Modular & Alta & Alta & Alta & $\uparrow$ \\
Relacional & Alta & Baja & Alta & $\downarrow$ \\
Jerarquía & Alta & Alta & Baja & Alta \\
\hline
\end{tabular}

Fuente: Gereffi, Humphrey y Sturgeon (2005: 87).

y la asimetría de poder. Éstas son inversamente proporcionales: cuando las transacciones necesitan más coordinación, el poder se reparte de manera más equilibrada entre los participantes, siendo el tipo ideal la transacción de mercado; en las transacciones en que la coordinación no es posible (o deseable), la toma de decisiones tenderá a concentrarse en las empresas líderes de la cadena de valor. Estas relaciones determinan qué actores concentran las mayores ganancias económicas al realizar las actividades que agregan más valor.

Las estructuras de gobernanza permiten conocer la articulación de la producción globalizada y los diferentes papeles que juegan los eslabones o nodos en la generación de valor. El estudio de estos elementos, establece las posibilidades que las redes de producción ofrecen para mejorar el desempeño y las condiciones de una integración exitosa en ellas.

A través de la acumulación de evidencia proporcionada por estudios de caso y de la reformulación constante de sus argumentos, esta teoría ha logrado establecer un conjunto de situaciones y de estrategias encaminadas a mejorar la participación de las empresas en general y de las economías dependientes en particular, en las cadenas de valor. Ello se expresa a través del concepto de ascenso industrial y de la caracterización del contexto institucional.

El diagnóstico acerca de los nichos o nodos en los que es posible integrarse a la cadena de valor y de las acciones que es preciso tomar para mejorar la posición con que se cuenta, constituye el proceso denominado ascenso industrial: "El ascenso industrial refiere el proceso por el cual actores económicos -naciones, firmas y trabajadores- se desplazan desde las actividades de bajo valor hacia las de relativamente alto valor en las redes de producción global. 
Diversas combinaciones de políticas gubernamentales, instituciones, estrategias corporativas, tecnologías y capacidades laborales, están asociadas al éxito del ascenso" (Gereffi, 2005: 171).

La trayectoria del ascenso industrial se determina a partir del grado de elaboración de los productos que aporta cada nodo de las cadenas. El eslabón inferior es la industria ensambladora que trabaja con insumos importados y por ello tiene muy escaso impacto en el desarrollo de las economías huéspedes; la manufactura de equipo original aporta mercancías genéricas utilizadas por diversos compradores; la manufactura de equipo de marca avanza en la diferenciación de los productos, adaptados a los requerimientos de los compradores; finalmente, la manufactura de diseño original incorpora las tareas más complejas, razón por la que los fabricantes son menos dependientes de sus compradores.

El contexto institucional juega un papel esencial para determinar cuáles son las condiciones que permiten transitar hacia los nodos que generan mayor valor agregado; las acciones y estrategias de las empresas y naciones para mejorar su posición en las cadenas de valor deben interactuar para lograr el ascenso industrial.

Dicho contexto institucional comprende:

- Las condiciones económicas, que refieren la disponibilidad de los principales insumos de la producción globalizada: niveles salariales, infraestructuras, financiamiento.

- El contexto social, que provee de trabajadores con la calificación requerida, y por ende, las instancias encargadas de su formación y capacitación.

- Las instituciones, que intervienen en diferentes terrenos: impulso del desarrollo científico y tecnológico, otorgamiento de subsidios, y puesta en marcha de regulaciones fiscales y laborales, entre los más importantes (Gereffi y Fernández-Stark, 2011: 11).

En suma, el enfoque de las cadenas globales de valor ha logrado sistematizar un amplio conjunto de argumentos para analizar la organización productiva, tanto dentro de las instituciones y actores económicos, como en sus relaciones al seno de las redes globalizadas de producción. También ha generado una extensa variedad de estudios de caso que aportan un conocimiento sin precedente acerca de las relaciones de producción capitalistas. Al ligar las posibilidades del desarrollo económico a la integración y ascenso en las cadenas de valor, este enfoque legitima la acción empresarial y propone encauzar la intervención estatal y del resto de actores económicos hacia la adaptación a 
las necesidades de las empresas líderes. El éxito económico e incluso la supervivencia de regiones y naciones quedan, de este modo, atados a la presencia y desempeños de las empresas y sus cadenas de producción.

\section{HACIA UNA ECONOMÍA POLÍTICA DE LA COMPETENCIA ENTRE GRANDES EMPRESAS}

El diálogo que proponemos con los cuerpos teóricos enunciados parte de establecer las diferencias en las perspectivas del análisis. Como contrapunto de lo hasta ahora expuesto, se trata de situar como categoría central el poder: cómo se forma, quién lo ejerce, qué consecuencias tiene en la reproducción de la sociedad capitalista, son las interrogantes centrales de este ejercicio. La economía política de la competencia busca explicar cómo se organizan las empresas transnacionales para producir mercancías, cómo se relacionan entre sí y con otros sujetos que participan en el mercado mundial, y cómo participan en la construcción de la hegemonía mundial.

Se parte de la concepción de la hegemonía como una construcción social que articula el ejercicio del poder. En la interpretación de Gramsci (1995), este concepto refiere la formación de una visión de mundo, aceptada universalmente, que expresa lo esencial de los intereses dominantes e incorpora parte de los intereses de las clases y los grupos subalternos. Extrapolada a la escala mundial, se define como la capacidad de crear y controlar las relaciones esenciales que permiten la reproducción del capitalismo. La hegemonía mundial se construye en cuatro planos principales: político-diplomático, militar, económico y cultural. Esta construcción es analizada a través de las estrategias y las prácticas del sujeto hegemónico, un sujeto desdoblado que comprende a los Estados y a las grandes corporaciones que disputan la hegemonía. ${ }^{4}$

En esta propuesta analítica, la competencia entre las grandes empresas articula la construcción hegemónica en su dimensión económica. Las corporaciones gigantes marcan las pautas de la reproducción del sistema y sus estrategias devienen el proyecto civilizatorio del capitalismo. Su impronta se expresa fundamentalmente a través de la organización productiva, pero no se limita a ese dominio: la acción empresarial desborda hacia todas las dimensiones de la vida social. Ello ocurre bajo la forma de un proceso de dominancia: las acciones de otros sujetos, y en particular las de los Estados, no desaparecen, 
pero quedan subordinadas a las estrategias y prácticas de las grandes corporaciones. Estos son los elementos de la propuesta que hemos denominado producción estratégica, la cual contrastamos con las teorías de la empresa analizadas en los apartados anteriores, buscando enriquecer la economía política de la competencia.

\section{Construcción del liderazgo económico mundial}

Para el estudio de la dimensión económica de la hegemonía, se propone el concepto de liderazgo económico mundial definido como la capacidad de las coaliciones de Estados y empresas para crear, desarrollar y controlar las fuentes de las ganancias, en relación al conjunto de empresas competidoras, particularmente en lo que toca a las formas de la organización productiva.

Esta definición marca las convergencias y diferencias con las propuestas teóricas dominantes en el análisis de la competencia. La organización productiva, el desarrollo científico y tecnológico, la producción globalizada y la nueva territorialidad creada por la competencia entre las empresas transnacionales, son temas comunes. La principal diferencia surge en torno a las motivaciones y los medios de la acción de las empresas. En tanto los enfoques dominantes consideran que la obtención de las ganancias y su aumento son resultado de una competencia en torno a las soluciones más eficientes, el enfoque de la producción estratégica considera los efectos de la concentración de diversas formas de poder en manos de las grandes empresas, de suerte que la acumulación de ganancias en pocas manos no es resultado sólo de la solución más eficiente, sino que es preciso tomar en cuenta también las prácticas monopólicas.

La diferencia es esencial ya que el argumento dominante otorga legitimidad y naturaliza la acción de las grandes empresas como la principal vía para alcanzar el desarrollo económico. En cambio, al tomar en cuenta las prácticas de poder, se reduce el alcance de la eficiencia como criterio ordenador de la economía capitalista, mostrando que las grandes empresas, al perseguir sus intereses particulares, no propician el bienestar general. Y aún más importante: las prácticas de poder revelan que la acción de las empresas transnacionales tiene consecuencias nefastas para las sociedades que las alojan y para el medio ambiente.

En esta perspectiva, se proponen cuatro instrumentos para el análisis de la competencia: 


\section{Capacidad de monopolización de las fuentes de ganancia}

La capacidad de monopolización es la base material del liderazgo económico; describe los medios con que cuentan las empresas derivados de la concentración de recursos de todo tipo: monetarios, tecnológicos, trabajadores, relaciones con instancias estatales y sociales, etcétera. Estos medios permiten a las empresas crear e imponer formas de organización productiva, y por esa vía, monopolizar las fuentes de las ganancias. La competencia se modela mediante la creación e imposición de normas productivas, impulsando las actividades de investigación y desarrollo, los acuerdos de regulación de la producción y reparto de mercados, la compra de empresas innovadoras o competidoras, las guerras de precios, las redes de subcontratación, etcétera. Hablamos de monopolio y no sólo de control, puesto que las estrategias de las grandes empresas construyen tanto las bases de su liderazgo como las condiciones de insuficiencia de sus competidores.

La escala de los recursos en manos de las empresas más grandes del mundo indica quién puede tomar decisiones con repercusiones globales, situación que se advierte en actividades como la explotación petrolera, la industria automotriz y la modificación genética de las semillas, en las que un pequeño grupo de empresas está marcando las pautas mundiales en sus respectivos mercados.

La concentración de poder en manos de las grandes empresas es una tendencia secular del capitalismo. La economía convencional intenta matizar, e incluso plantear como superada, dicha tendencia; mientras que nuestro estudio de la competencia indica que no sólo sigue vigente, sino que se ha acentuado en los años recientes: el capitalismo del siglo Xxi puede ser caracterizado como la sociedad modelada por las grandes corporaciones.

\section{Vanguardia tecnológica}

La vanguardia tecnológica es definida como la capacidad de crear nuevas tecnologías en las actividades esenciales para la reproducción del capitalismo, particularmente en los campos de frontera y de uso generalizado. Se trata de analizar las innovaciones que crean nuevas formas de producir mercancías y aquellas que afectan al conjunto de la economía.

Las jerarquías que surgen de la capacidad de monopolización son afectadas por las innovaciones. La teoría económica considera que la tecnología juega un papel disruptivo en el proceso económico, aumentando las posibilidades de la generación de ganancias y poniendo en cuestión la posición de las em- 
presas líderes. Es necesario añadir que, en el marco de una sólida dominación económica, las innovaciones también pueden reforzar las jerarquías existentes al ser apropiadas por las grandes empresas. El alcance de las innovaciones está ligado a la concentración de recursos: no todos los capitales tienen la capacidad de crear tecnologías disruptivas en amplias escalas.

A través de estas prácticas, las grandes empresas construyen las vías de evolución de las actividades en que actúan, modelándolas a sus necesidades, con el objetivo central de alcanzar la mayor rentabilidad posible.

\section{Estrategias y prácticas de "cooperación" entre empresas}

En condiciones de equilibrio relativo entre los participantes, la competencia adquiere formas "cooperativas" en las que empresas rivales acuerdan desarrollar una tecnología, un producto, o controlar alguna fuente de aprovisionamiento. Estas formas "cooperativas" tienen horizontes prácticos y temporales acotados. Las empresas crean instancias de cooperación entre ellas y con los Estados u otros sujetos: alianzas tecnológicas, empresas conjuntas, consorcios público-privado para desarrollar algún sector o actividad, etcétera.

Entre estas prácticas destacan dos: las alianzas tecnológicas, que buscan hacer frente a los crecientes costos de la innovación en las actividades controladas por las corporaciones gigantes; $y$ los consorcios público-privado para fomentar la investigación y el desarrollo, que permiten que los acervos de financiamiento y de conocimientos en manos de instancias estatales e instituciones educativas fluyan hacia las empresas y mejoren sus procesos de producción.

Estas prácticas "cooperativas" han suscitado una amplia polémica acerca de la vigencia de la competencia como principal forma de relación entre las empresas, en tanto la disputa directa es desplazada por relaciones basadas en la negociación y el acuerdo. La cuestión esencial reside en el tipo de producto, especialmente, el tipo de tecnología, que se elabora en forma conjunta. En el caso de las grandes empresas, se advierte que más allá del mutuo beneficio para los participantes en la cooperación, persisten las asimetrías de poder y de aprovechamiento de los resultados, siempre favorables a las grandes corporaciones.

\section{Vectores de la acción estatal}

El Estado constituye uno de los nodos esenciales de la competencia, ya que realiza dos tareas estratégicas para la reproducción del capitalismo:

En primer lugar, en tanto espacio que procesa los conflictos sociales, el Estado formula y realiza el interés general, en dos vertientes principales: la lectura 
estratégica de las posibilidades que enfrenta el conjunto económico bajo su jurisdicción (qué actividades impulsar, con qué objetivos), y la negociación de las políticas y acciones estatales con las fracciones más poderosas del empresariado. En ello se juegan la legitimidad, la gobernabilidad y la coherencia del funcionamiento económico.

En segundo lugar, el Estado debe crear y mantener las condiciones que hacen posibles los mercados: el establecimiento de las reglas del juego para la competencia, y las políticas y prácticas que permiten la continuidad de la explotación de los trabajadores y de las riquezas naturales.

Algunas prácticas estratégicas a través de las cuales el Estado interviene en la competencia son:

El Estado como inversionista. Actualmente las inversiones por parte de los Estados revisten un carácter esencial en tanto van destinadas a proveer capital, bienes, servicios o personal calificado que las empresas no pueden aportar. El capital de riesgo (a menudo a fondo perdido) es una forma típica de la intervención estatal, en tanto financia la experimentación de mejoras productivas, tarea que no es rentable para la mayoría de las empresas.

Creación de marcos regulatorios. La obtención de ganancias se garantiza mediante la formulación de fronteras para las actividades económicas y reglas de funcionamiento para los mercados. Ejemplos de ello son las legislaciones sobre política de competencia, propiedad intelectual e inversiones extranjeras. Estos son campos en que el "arbitraje" del Estado se impone como la solución menos onerosa, pues el acuerdo entre grandes capitales resulta no sólo costoso si no a menudo imposible de alcanzar.

Estímulos directos a la producción capitalista. Se trata del otorgamiento de subsidios, concesiones de terrenos, exenciones de pagos, régimen fiscal, etcétera. Esta es la forma de intervención "indirecta” más conocida: el Estado entrega parte del excedente económico a las empresas, y en la mayoría de los casos, lo hace en beneficio de las grandes empresas.

Realización y fomento del progreso cientifico y tecnológico. El Estado destina fondos públicos a la investigación y desarrollo, complementando el esfuerzo tecnológico de las entidades privadas. Estos aportes se dan mediante el financiamiento de las instituciones de educación e investigación y a través de instancias estatales dedicadas a innovar, como es el caso de los laboratorios militares estadounidenses, origen de buena parte de las innovaciones cruciales en los últimos 75 años.

Politicas y acciones de control social. En razón de la creciente polarización social, los Estados realizan enormes gastos en medios de control social. Los más conocidos son los dedicados a los medios de represión abierta: fortaleci- 
miento de las fuerzas armadas, compra de armamento, de servicios y equipos de vigilancia y espionaje, etcétera. A ello se suman los gastos en asistencia social y promoción electoral, así como los gastos aparentemente suntuarios, en espectáculos masivos y campañas de imagen.

El dispositivo de intervención estatal en la competencia es multidimensional y no está determinado por la eficiencia o el "interés general”, sino por los resultados de la negociación con los sectores más poderosos del empresariado, siendo influenciado también por las presiones de los sujetos dominados.

Estas son las dimensiones más importantes de la competencia. Además de los aspectos relativos a la organización productiva y las intervenciones institucionales, el enfoque de la producción estratégica coloca la construcción del liderazgo económico mundial como la relación que articula al conjunto de estrategias y prácticas de los sujetos que participan en la competencia.

\section{CONCLUSIONES}

Este trabajo ha propuesto establecer un diálogo con las teorías dominantes en el estudio de la competencia entre las grandes empresas, con el fin de mejorar la herramientas teóricas y metodológicas, y por esa vía, conocer el comportamiento de estos importantes agentes, principales organizadores de la economía contemporánea.

Los enfoques de los costos de transacción y de las cadenas de valor avanzan en el análisis de la organización productiva, y proponen políticas para lograr una integración más eficiente a la economía mundial. A lo largo del artículo, se han detallado esos aportes, buscando incorporarlos a la lectura inicial de la competencia capitalista a partir de las relaciones de poder. Para ello, se hizo una lectura crítica de dichos enfoques, buscando enriquecer la economía política de la competencia.

Este diálogo ha permitido establecer elementos comunes, como son: la centralidad de las empresas líderes en la articulación del mercado mundial, la importancia de la organización de la producción que tales empresas llevan a cabo, el papel transformador del desarrollo tecnológico y la necesidad de considerar las instituciones que, además de las empresas, participan en el proceso económico, aspectos que no siempre son tomados en cuenta en los análisis críticos.

El concepto de liderazgo económico mundial retoma los resultados de este ejercicio teórico, en tanto sintetiza las relaciones entre empresas, mercados e instituciones, permitiendo analizar la competencia entre grandes empresas 
en el mercado mundial y sus posibles evoluciones. En él, la cuestión de la eficiencia sigue siendo importante pero, a diferencia de lo que proponen las teorías dominantes, queda subordinada a las relaciones de liderazgo: monopolización, jerarquía, vanguardia tecnológica. De este modo, se formula una metodología que permitirá análisis más complejos de la competencia entre las grandes empresas transnacionales.

\section{BIBLIOGRAFÍA}

Bair, Jennifer (2008), "Global Commodity Chains: Genealogy and Review", en Jennifer Bair (ed.), Frontiers of Commodity Chain Research, Palo Alto, Stanford University Press, pp. 1-34.

Cantwell, John (1991), "A Survey of Theories of International Production", en Christos Pitelis y Roger Sugden (ed.), The Nature of de Transnational Firm, New York, Routledge, pp. 10-56.

Ceceña, Ana Esther (2004), "Estrategias de construcción de una hegemonía sin límites", en Ana Esther Ceceña (comp.), Hegemonias y emancipaciones en el siglo XXI, Buenos Aires, ClACso, pp. 37-58.

Ceceña, Ana Esther y Andrés Barreda (coords.) (1995), Producción estratégica y hegemonía mundial, México, Siglo Xxi Editores, 541 pp.

Coase, Ronald H. (1937), "The Nature of the Firm”, Economica, New Series, vol. 4, núm. 16, London, pp. 386-405.

Coriat, Benjamin y Oliver Weinstein (2011), Nuevas teorias de la empresa. Una revisión crítica, Buenos Aires, Lenguaje claro Editora, 222 pp.

Foss, Nicolai J. (2011), The Theory of the Firm. Critical Perspectives on Business and Managment, London, Routledge, 4 volúmenes.

Gramsci, Antonio (1995), "Americanismo y fordismo", Cuadernos de la cárcel 1: Notas sobre Maquiavelo, sobre politica y sobre el Estado moderno, México, Juan Pablos, pp. 281-317.

Gereffi, Gary (1994), "The Organization of Buyer-Driven Global Commodity Chains: How U.S. Retailers Shape Overseas Production Networks", en Gary Gereffi y Miguel Korzeniewicz (eds.), Commodity Chains and Global Capitalism, Westport, Greenwood Publishing Group, pp. 95-122.

(2001), "Las cadenas productivas como marco analítico para la globalización”, Problemas del Desarrollo, vol. 32, núm. 125, México, IIEc-Unam, abril-junio, pp. 9-37.

(2005), "The Global Economy: Organization, Governance, and Development", Neil J. Smelser y Richard Svedberg (eds.), The Handbook of Economic Sociology, Princeton, Princeton University Press, pp. 160-182. 
Gereffi, Gary y Karina Fernández-Stark (2011), Global Value Chains Analysis: A Primer, North Carolina, Center on Globalization, Governance \& Competitiveness, $40 \mathrm{pp}$.

, John Humphrey y Timothy Sturgeon (2005), "The Governance of Global Value Chains", Review of International Political Economy, 12:11, Routledge, febrero, pp. 78-104. DOI: 10.1080/09692290500049805. y Miguel Korzeniewicz (eds.) (1994), Commodity Chains and Global Capitalism, Westport, Greenwood Publishing Group, 344 pp.

, Miguel Korzeniewicz y Roberto Korzeniewicz (1994), "Introduction: Global Commodity Chains", en Gary Gereffi y Miguel Korzeniewicz (eds.), Commodity Chains and Global Capitalism, Westport, Greenwood Publishing Group, pp. 1-14.

Hopkins, Terence e Immanuel Wallerstein (1986), "Commodity Chains in the World-economy Prior to 1800”, Review, 10:1, pp. 157-170.

Hymer, Stephen (1972), Empresas multinacionales: La internacionalización del capital, Buenos Aires, Periferia, 174 pp.

Kindleberger, Charles P. (1969), American Business Abroad. Six Lectures on Direct Investment, New Haven, Yale University Press, 225 pp.

Marshall, Alfred (2005), Principios de economía, Madrid, Síntesis, 2 volúmenes.

Pitelis, Christos N. y Roger Sugden (2000), The Nature of the Transnational Firm, London, Routledge, 220 pp.

Porter, Michael (1991), La ventaja competitiva de las naciones, Buenos Aires, J. Vergara, 1025 pp.

Stacey, Frederik (2016), Concepts \& Tools, Durham, Duke University, Center on Globalization, Governance \& Competitiveness (consultado el 10 de septiembre de 2016), disponible en <https:/globalvaluechains.org/ concept-tools>

Sturgeon, Timothy (2008), "From Commodity Chains to Value Chains. Interdisciplinary Theory Building in an Age of Globalization", en Jennifer Bair (ed.), Frontiers of Commodity Chain Research, Palo Alto, Stanford University Press, pp. 110-135.

Williamson, Oliver E. (1989), Las instituciones económicas del capitalismo, México, Fondo de Cultura Económica, 435 pp. 